\title{
A Novel Two-Dimensional Readout Design for Floating Strip Micromegas Detectors
}

\author{
Felix Klitzner, ${ }^{a, *}$ Otmar Biebel, ${ }^{a}$ Jonathan Bortfeldt, ${ }^{a}$ Bernhard Flierl, ${ }^{b}$ Maximilian \\ Herrmann $^{a}$ and Ralf Hertenberger ${ }^{a}$ \\ ${ }^{a}$ Ludwig-Maximilians-University, \\ Am Coulombwall 1, Munich, Germany \\ ${ }^{b}$ ENVINET GmbH, \\ Hans-Pinsel-Str. 4, Munich, Germany \\ E-mail: felix.klitzner@physik.uni-muenchen.de
}

\begin{abstract}
Floating strip Micromegas detectors are high-rate capable particle detectors with excellent spatial and time resolution, allowing single particle tracking with fluxes up to at least $7 \mathrm{MHz} / \mathrm{cm}^{2}$. The amplified ionization charge is collected on copper anode strips kept at a slightly floating electrical potential. The charge signal is coupled to two layers of perpendicular readout strips in $\mathrm{x}$ and $\mathrm{y}$, insulated by thin Kapton layers. A complete understanding of the signal formation in the detector, particularly on the orthogonal y-strips, is achieved: simulations disentangle the signal coupling due to alternating weighting field lines with respect to the ion velocity vector and the response of charge sensitive front-end electronics. Different two-dimensional anode designs with optimized strip geometry have been developed based on the simulations. We present the performance of the detector for the different designs measured in $20 \mathrm{MeV}$ proton/neutron and $180 \mathrm{GeV}$ pion/muon beams, using APV25 front-end electronics.
\end{abstract}

40th International Conference on High Energy physics - ICHEP2020

July 28 - August 6, 2020

Prague, Czech Republic (virtual meeting)

\footnotetext{
${ }^{*}$ Speaker
} 


\section{Introduction}

One-dimensional floating strip Micromegas [1][2] are high-rate capable gaseous particle detectors with excellent spatial and temporal resolution, allowing single particle tracking for particle fluxes up to $7 \mathrm{MHz} / \mathrm{cm}^{2}$ [2]. Ionization charge is generated in the typically $6 \mathrm{~mm}$ wide drift region between cathode and mesh. The copper anode strips are individually connected

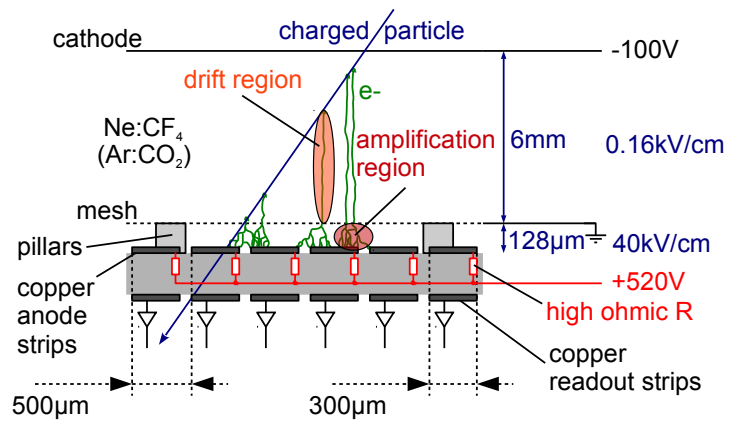

Figure 1: Scheme of a floating strip Micromegas with one-dimensional readout. to high-voltage via high-ohmic resistors. After gas amplification, the signal is collected on these floating anode strips and capacitively coupled to copper readout strips (see fig. 1), insulated by a thin Kapton layer. This scheme of 'floating' anode strips strongly suppresses the sensitivity of the detector to discharges between mesh and anode, induced by e.g. strongly ionizing particles. A two-dimensional prototype readout design with two layers of copper readout strips in $\mathrm{x}$ and $\mathrm{y}$ has been developed and successfully tested [3]. Due to different coupling processes of the signal generated in the amplification region to the two readout strip layers, novel anode strip structures have been designed based on simulations and intensively tested in various beam environments [4].

\section{Signal Formation in Two-Dimensional Floating Strip Micromegas}

The coupling of the signal generated in the amplification gap to the strips of the anode is a combination of two processes: The directly induced currents on the strips due to moving charges in the avalanche and the capacitive coupling of these currents between strips. The directly induced current on an electrode $i$ of a moving charge $q$ can be calculated via weighting fields, following the Shockley RamoTheorem [5]

$$
I_{i}^{\text {ind }}(t)=-q / V_{w} \cdot \mathbf{E}_{i}[\mathbf{x}(t)] \cdot \mathbf{v}(t)
$$

where $\mathbf{E}_{i} / V_{w}$ is called the weighting field of

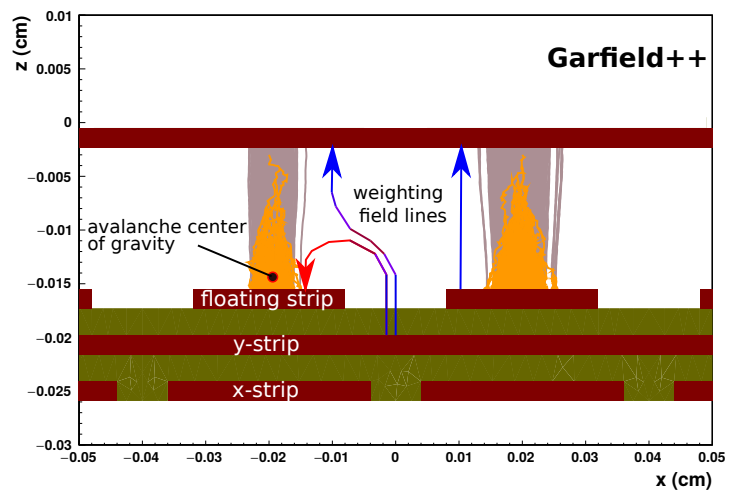

Figure 2: Simulation of two avalanches in the amplification region with two-dimensional readout. The most important weighting field lines are sketched. electrode $i$ and $\mathbf{v}(t)$ the velocity vector of the moving charge. The electric fields in a two-dimensional floating strip Micromegas have been analytically calculated by ANSYS and are imported into GARFIELD++ to simulate the charge carrier drift and amplification (see fig. 2). As during the amplification process the velocity vector of the electrons (ions) is always anti-parallel (parallel) to the weighting field lines of the floating strips, the current induced by the movement of the electrons towards the strips and the ions towards the mesh is unipolar negative. On the perpendicular yreadout strip however, the induced current is first positive and then negative, depending on the position of the charge during the amplification process: As most of the charge is created close to the 
anode strips, the induced electron signal is almost exclusively positive. The ion drift towards the mesh however initially induces a positive signal when the ions are close to the anode strips and a negative current signal after crossing approximately the middle of the amplification gap, where the weighting field lines bend towards the micro-mesh. The induced currents are simultaneously shared with surrounding electrodes by capacitive coupling. The capacitances inside the detector have been calculated with ANSYS and are used for the charge sharing calculation. The final current signal on the anode readout strips is then a superposition of directly induced and capacitively coupled currents as can be seen in fig. 3. While for the $\mathrm{x}$-strips the unipolar negative current signal is dominantly
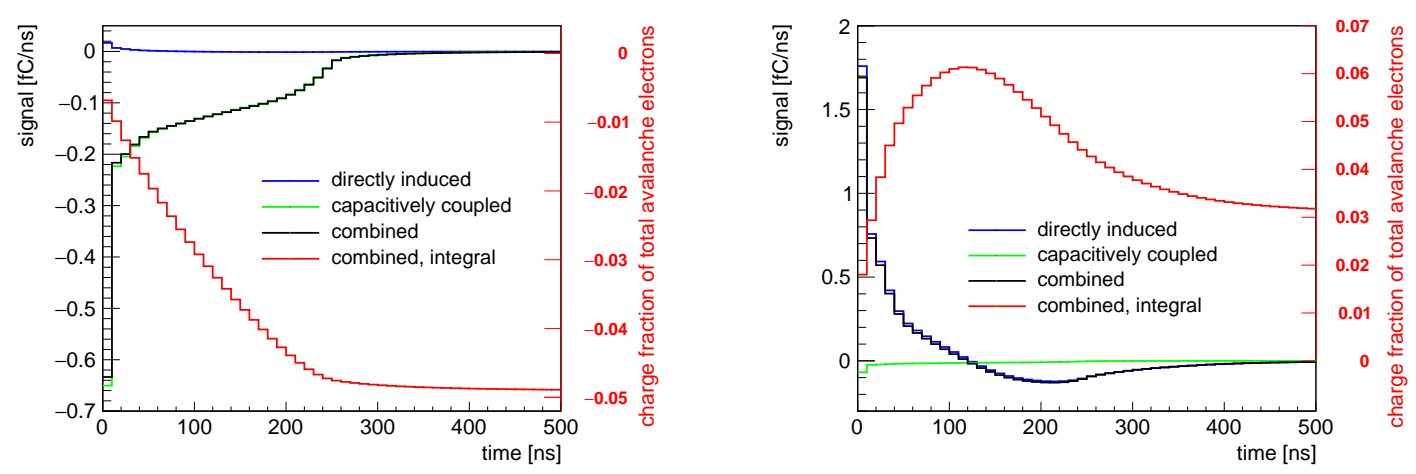

Figure 3: Simulated current on the $x$-strip (left) and on the y-strip (right) as a function of time. The different contributions from directly induced and capacitively coupled signals are visible.

created by capacitive coupling from the congruent floating strips, the y-strip signal is dominantly created by the directly induced bipolar, initially positive current signal. To validate the simulation, the currents created from the passage of a cosmic muon have been convoluted with a simplified transfer-function of a charge sensitive preamplifier, followed by a CR-RC-shaping stage (see fig 4). Finite front-end board input impedance as well as strip capacitance are taken into account. The good agreement between simulation and measurement is visible.
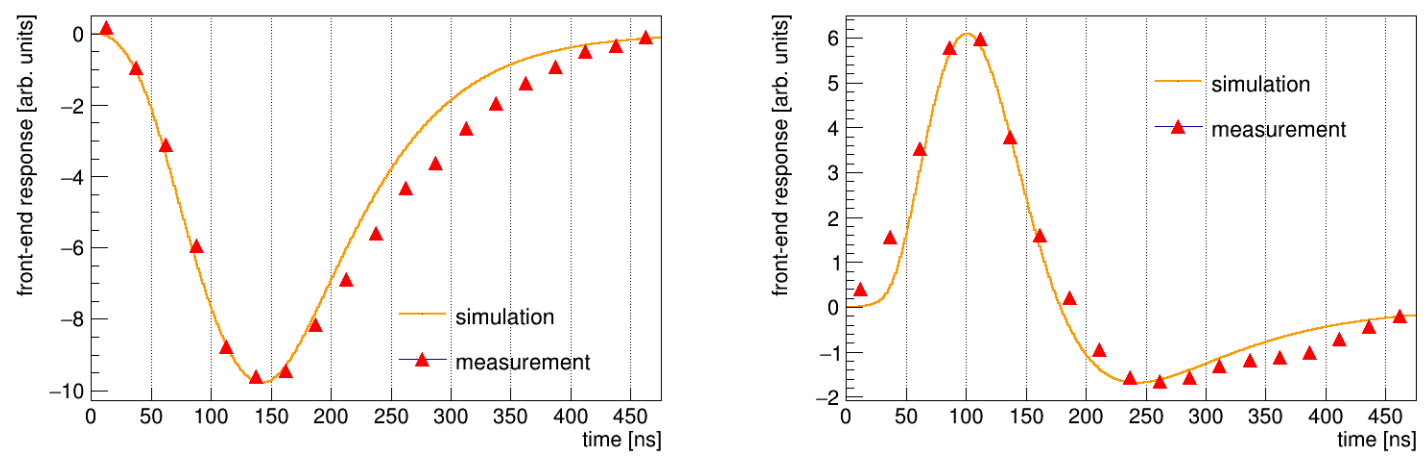

Figure 4: Simulated and measured front-end response of a cosmic muon traversing the detector with an APV-25 hybrid board on the x-strip (left) and the y-strip (right).

As the total charge coupled to both readout strips is smaller than $6 \%$ of the charge created in the amplification process, an optimized anode strip layer geometry was developed. 


\section{A Novel Two-Dimensional Readout Strip Design with Optimized Strip Geometry}

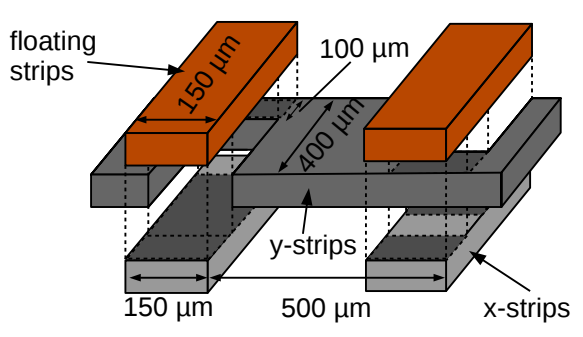

Figure 5: Novel optimized strip layer setup: The y-strip features an alternating pattern.

In order to improve the signal yield on both readout strip layers, a novel two-dimensional anode PCB has been developed. Fig. 5 shows the strip layer setup with $35 \mu \mathrm{m}$ thick copper strips which are insulated by $25 \mu \mathrm{m}$ thick Kapton layers (not drawn). To maximize the directly induced signal on the y-strips they are placed under the floating strips, which feature a small width-to-pitch ratio of $150 / 500$, with a maximized width in between the floating strips. To avoid creating large capacitances to the $y$-strips and at the same time increasing the capacitive coupling between floating and $\mathrm{x}$-strips, the width of the y-strips is minimized below the floating strips. The simultaneous increase in signal amplitude on $\mathrm{x}$ - and y-strip is visible in fig. 6, where a classic anode design with straight strips is compared to the novel one, both measured with an ${ }^{55} \mathrm{Fe}$-source under controlled identical environmental conditions using $\mathrm{Ne}: \mathrm{CF}_{4} 80: 20$ vol. \% as detector gas.
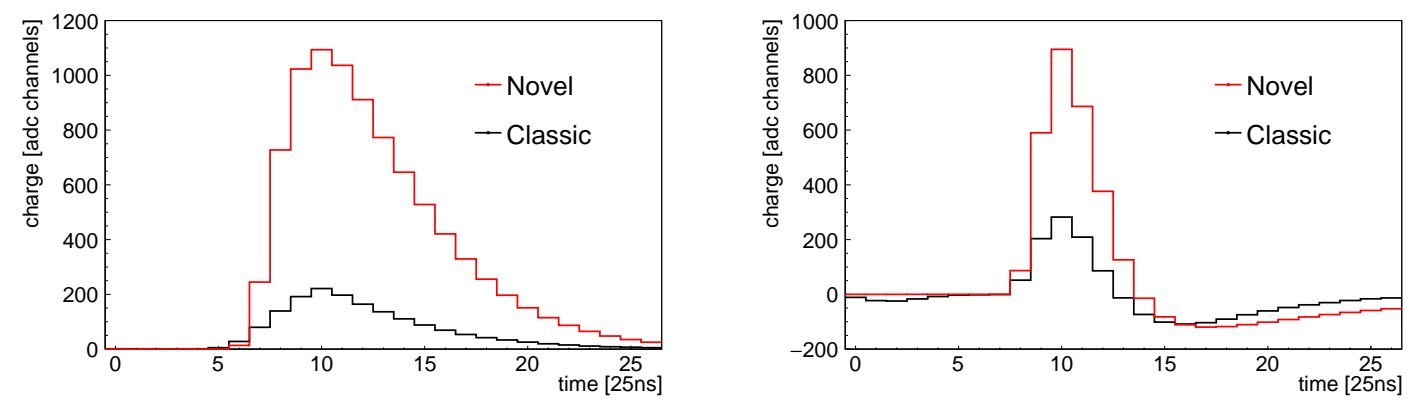

Figure 6: Average signal on the strip with maximum charge for the x-layer (left) and the y-layer (right) with a classic design (black) and the novel design (red) measured with a ${ }^{55} \mathrm{Fe}$-source.

\section{Performance}

To investigate the particle reconstruction performance of a two-dimensional floating strip Micromegas with the optimized anode strip layer geometry, it has been tested with minimum (MIP) and highly ionizing particles, using Ne:CF $80: 20$ vol. \% as detector gas. During the tests with MIPs the spatial resolution and efficiency has been determined with rates up to almost $5 \mathrm{MHz} / \mathrm{cm}^{2}$. Tests with neutrons at a rate of several $\mathrm{kHz} / \mathrm{cm}^{2}$ and a ${ }^{10}$ Boron coated cathode confirm the highvoltage stability of the detector even at MIP gain values in harsh, densely ionizing background environments.

\subsection{Measurements with Muons and Pions at the CERN SPS H8 Beamline}

The spatial resolution and efficiency is investigated using four resistive strip Micromegas with 2d-hit information as an external track reference. The hits in the Micromegas are reconstructed using the so-called centroid method which calculates the charge center of gravity position for a cluster of strips. The residual distribution between track prediction and measured hit position of both $\mathrm{x}$ - 
and y-strips of the floating strip Micromegas are shown in fig. 7. A double Gaussian fit extracts
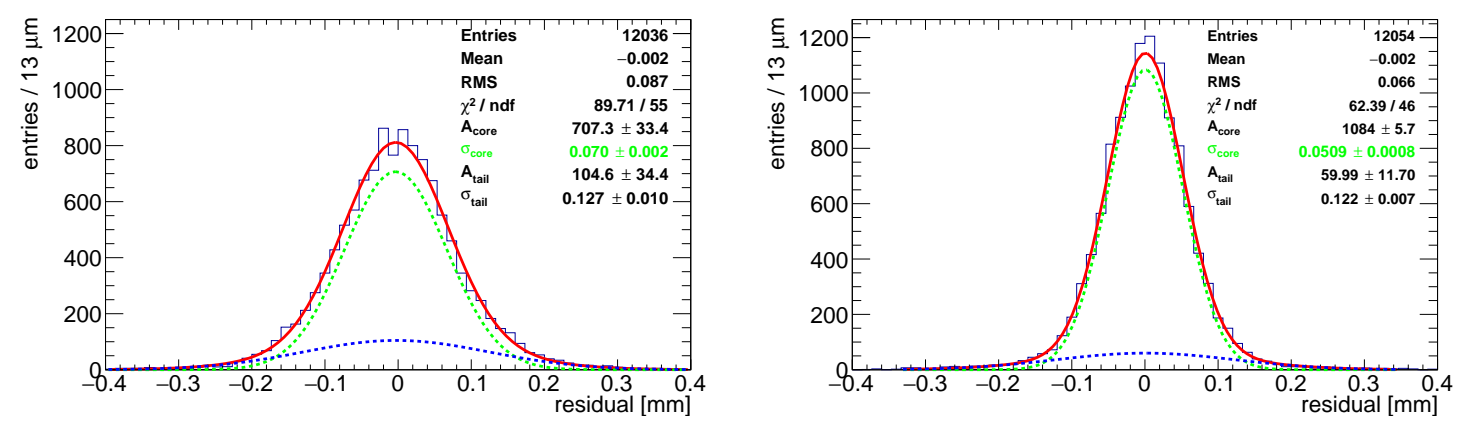

Figure 7: Exclusive residual of the $\mathrm{x}$-strips (left) and $\mathrm{y}$-strips (right), measured with the optimized anode design at a perpendicular incident pion beam at a rate of $420 \mathrm{kHz} / \mathrm{cm}^{2}$.

the weighted spatial resolution between core and tail distributions. Unfolding the track accuracy of $23 \mu \mathrm{m}$ from the combined residual width, a spatial resolution of $(79 \pm 4) \mu \mathrm{m}$ for the $\mathrm{x}$-strips and $(54 \pm 2) \mu \mathrm{m}$ for the $\mathrm{y}$-strips is found. The $\mathrm{x}$-strips resolution is limited by charge discretization on the floating strips as the lateral ionization charge cloud size is of the same order as the anode strip pitch. An efficiency of $(98.0 \pm 0.2) \%$ in a $5 \sigma$ window around the Gaussian distributions is found. The spatial resolution of $\mathrm{x}$ - and $\mathrm{y}$-strips as a function of the particle inclination angle benefits from the so called $\mu$ TPC-method, which makes use of the signal timing rather than the charge. While for the centroid method the spatial resolution degrades up to almost $600 \mu \mathrm{m}$ at $40^{\circ}$ incidence, the $\mu$ TPC-method stays below $150 \mu \mathrm{m}$ when using relatively small drift fields with electron drift velocities around $(30 \pm 2) \mu \mathrm{m} / \mathrm{ns}$. The efficiency stays above $97 \%$ when choosing amplification fields above $48 \mathrm{kV} / \mathrm{cm}$, see further details in [4]. To check the detector performance at high rates, the pion beam flux under perpendicular incidence was increased up to $4.7 \mathrm{MHz} / \mathrm{cm}^{2}$. This leads to an increased probability to record secondary fragments of pion-nucleus interactions in a single triggered event. Thus an iterative Kalman tracking algorithm was used to identify the correct cluster combinations along the detector telescope. The cluster charge as well as the tracking efficiency of $\mathrm{x}$ - and $\mathrm{y}$-strips of the floating strip Micromegas as a function of the beam flux is shown in fig. 8. The
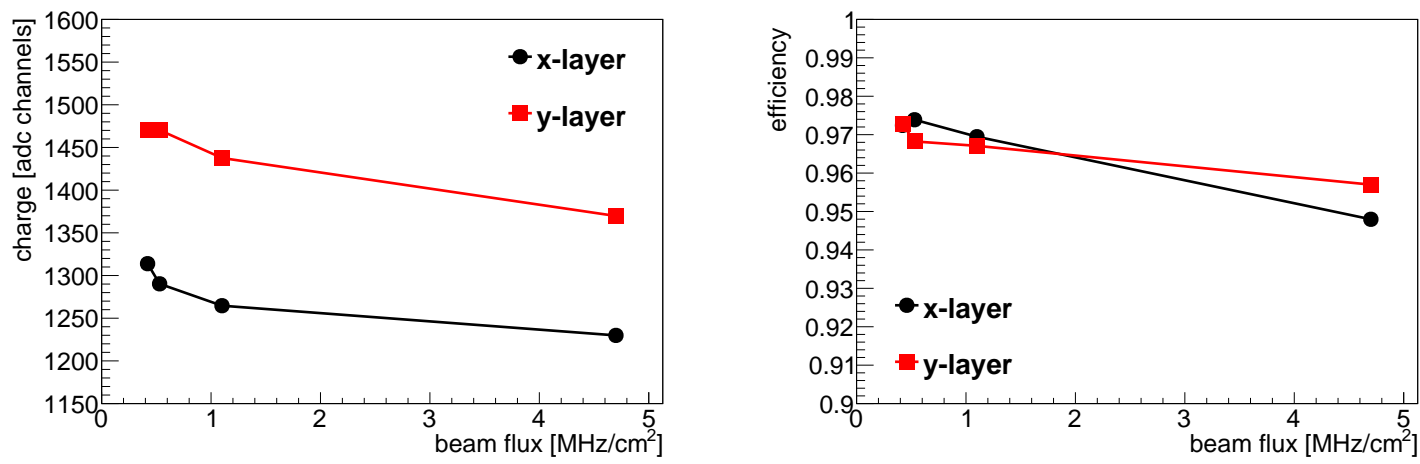

Figure 8: Mean cluster charge (left) and $5 \sigma$ efficiency (right) as a function of the beam flux.

efficiency stays above $95 \%$ for both strip layers despite the expected cluster charge drop of around $10 \%$ for the highest measured rate point, which is a direct consequence of a gain drop resulting from a voltage drop of $5 \mathrm{~V}$ when recharging the floating strips over the $22 \mathrm{M} \Omega$ resistors. 


\subsection{Measurements with Neutrons at the MLL Tandem Accelerator in Garching}

The floating strip Micromegas high-voltage stability is tested under $\mathrm{kHz} / \mathrm{cm}^{2}$ Neutron irradiation in combination with a ${ }^{10} \mathrm{~B}$ coated cathode. In the interaction process the neutron is captured in the cathode and breaks the ${ }^{10} \mathrm{~B}$ atom into a Lithium and Helium ion. Fig. 9 (top) shows the Boron coated cathode with a copper tape structure and the reconstructed neutron conversion point (bottom). The binned structure in the neutron hit distribution originates from using the latest responding strip in both dimensions to reconstruct the conversion position at the cathode, thus leading to a strip discretized hit information. The stopping of Lithium and Helium ions in the copper tape is clearly visible. Due to the high energy loss of the fragments, neutrons create very high signals in the Micromegas. Under MIP settings with gain values of around $10^{4} \mathrm{a}$ stable operation is observed despite the anode drawing constant currents of around $7 \mu \mathrm{A}$.

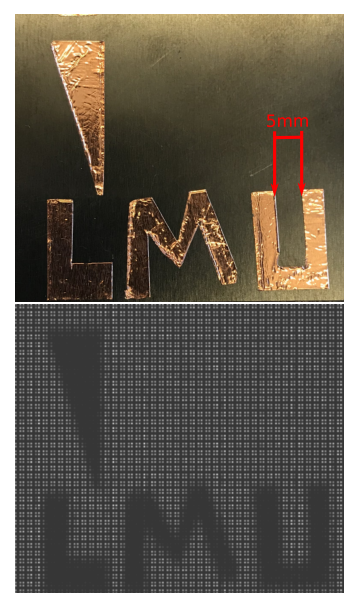

Figure 9: ${ }^{10} \mathrm{~B}$ coated cathode with copper tape (top). Measured neutron conversion point at the cathode (bottom).

\section{Summary}

We have presented a novel two-dimensional readout design for a floating strip Micromegas. The raw signal is increased by at least a factor of two compared to classical anode designs. Optimum spatial resolution of $(79 \pm 4) \mu \mathrm{m}$ for the $\mathrm{x}$-strips and $(54 \pm 2) \mu \mathrm{m}$ for the $\mathrm{y}$-strips are found at an efficiency of $(98.0 \pm 0.2) \%$, which stays almost constant up to beam fluxes of $4.7 \mathrm{MHz} / \mathrm{cm}^{2}$. A stable operation at high gain values of $10^{4}$ under $\mathrm{kHz} / \mathrm{cm}^{2}$ Neutron bombardment is observed.

\section{Acknowledgments}

We acknowledge the support by the DFG Excellence Cluster 'Origin and Structure of the Universe' and the crew of the MLL and CERN-SPS-H8 beamline.

\section{References}

[1] Y. Giomataris et al., Micromegas: a high-granularity position sensitive gaseous detector for high particle-flux environments, Nucl. Instr. Meth. A 37629 - 35, 1996.

[2] J. Bortfeldt, The Floating Strip Micromegas Detector: Versatile Particle Detectors for Highrate Applications (Springer Theses), Springer, ISBN 978-3-319-18892-8, 2015.

[3] J. Bortfeldt et al., Low material budget floating strip Micromegas for ion transmission radiography, Nucl. Instr. Meth. A, ISSN 0168-9002, https://dx . doi .org/10 .1016/j . nima. $2016.05 .003,2016$.

[4] F. Klitzner, Novel Two-Dimensional Floating Strip Micromegas Detectors, LAP LAMBERT Academic Publishing, ISBN 978-620-0-45646-5, 2019.

[5] W. Shockley, Currents to Conductors Induced by a Moving Point Charge, Journal of Applied Physics, 9(10):635-636, https://doi .org/10 .1063/1.1710367, 1938. 